\title{
Mycobacterium abscessus - an uncommon, but important cause of peritoneal dialysis- associated peritonitis - case report and literature review
}

\author{
Anup Singh Jheeta ${ }^{1}$, Jayakeerthi Rangaiah², John Clark², David Makanjuola ${ }^{1}$ and Subash Somalanka ${ }^{{ }^{*}}$ (D)
}

\begin{abstract}
Background: Peritoneal dialysis (PD) is a form of therapy for end-stage kidney disease (ESKD), and peritonitis is a known complication. Mycobacterium (M) species associated peritonitis in PD patients is uncommon. Our experience of managing PD associated peritonitis caused by M abscessus in a middle-aged man with ESKD due to focal segmental glomerulosclerosis is shared in this article with a review of the literature on this condition.

Case presentation: A 49-year old man presented to our unit with symptoms of peritonitis and cloudy PD effluent. Initial analysis of PD fluid showed Gram stain was negative, with no organism grown. Empirical PD peritonitis treatment with intra-peritoneal antibiotics did not improve his symptoms and he required intravenous antibiotics, PD catheter removal and a switch to haemodialysis. Cultures of the PD fluid later grew M abscessus, and the antibiotic regimen was changed appropriately, leading to clinical improvement.

Conclusion: $M$ abscessus associated peritonitis in PD patients is rare. It needs to be borne in mind when clinical improvement is not seen with standard broad-spectrum antibiotics, especially in situations where the PD fluid is initially deemed to be culture negative. PD fluid samples should be sent for acid-fast bacillus and if detected, should be further analysed with genome-wide sequencing to confirm the species of the Mycobacterium. Prompt removal of the catheter with peritoneal washout is critical for clinical improvement.
\end{abstract}

Keywords: Peritoneal dialysis, Catheter, Peritonitis, Mycobacterium abscessus

\section{Background}

Peritoneal dialysis is used for managing end-stage kidney disease (ESKD). It requires access to the peritoneal space by way of a percutaneous tunnelled dialysis catheter. Infective complications of peritoneal dialysis (PD) include catheter exit-site infection (ESI), catheter tunnel infection (TI) and peritonitis. Peritonitis can be particularly

\footnotetext{
* Correspondence: Nephr0nite@gmail.com

'South West Thames Renal \& Transplantation Unit, Epsom \& St Helier University Hospitals NHS Trust, Wrythe Lane, Carshalton, Surrey SM5 1AA, UK Full list of author information is available at the end of the article
}

severe and can lead to PD failure and discontinuation of PD. [1] Early recognition of infections, prompt microbiological diagnosis, and establishing appropriate treatment are therefore essential. An essential factor that determines a successful outcome in such cases is antimicrobial susceptibility testing to guide appropriate therapy. PD related peritonitis is most commonly caused by gram-positive aerobes such as coagulase-negative Staphylococci and Staphylococcus aureus, or gramnegative aerobes such as Pseudomonas aeruginosa [2]. Reports of PD related peritonitis caused by Mycobacterium species are relatively rare, although their incidence

(C) The Author(s). 2020 Open Access This article is licensed under a Creative Commons Attribution 4.0 International License, which permits use, sharing, adaptation, distribution and reproduction in any medium or format, as long as you give appropriate credit to the original author(s) and the source, provide a link to the Creative Commons licence, and indicate if changes were made. The images or other third party material in this article are included in the article's Creative Commons licence, unless indicated otherwise in a credit line to the material. If material is not included in the article's Creative Commons licence and your intended use is not permitted by statutory regulation or exceeds the permitted use, you will need to obtain permission directly from the copyright holder. To view a copy of this licence, visit http://creativecommons.org/licenses/by/4.0/. The Creative Commons Public Domain Dedication waiver (http://creativecommons.org/publicdomain/zero/1.0/) applies to the data made available in this article, unless otherwise stated in a credit line to the data. 
has been well documented [3, 4]. Nontuberculous mycobacteria (NTM) are members of the genus Mycobacterium but are significantly different from other members of the same genus, e.g., M.tuberculosis and M.leprae. NTM related PD peritonitis is emerging as a diagnostic and therapeutic challenge in the management of $\mathrm{PD}$ peritonitis, with comparatively higher rates of PD catheter loss and PD failure compared to other organisms [5]. Consensus on the management of NTM related PD peritonitis does not currently exist, possibly because of the paucity of reported cases. We describe what is to our knowledge the first reported case in the UK of PD related peritonitis secondary to the NTM species Mycobacterium abscessus.

\section{Case presentation}

A 49-year-old male of Afro-Caribbean origin with a background of hypertension, type 2 diabetes mellitus, high body mass index of 40 and ESKD secondary to focal and segmental glomerulosclerosis (FSGS) diagnosed in 2007 and received only 1 month of corticosteroids. He presented to our PD unit with mild abdominal discomfort and cloudy PD effluent in July 2019. He had been on PD for 4 years and had experienced one previous episode of PD peritonitis caused by Staphylococcus aureus requiring catheter replacement 2 years ago. The PD regimen consisted of automated peritoneal dialysis [APD] of $8.5 \mathrm{~h}$ duration with 5 cycles of 31 fills. The dialysate fluid consisted of $1.36 \%$ glucose concentration of 101 and $2.27 \%$ glucose concentration of 51 with extraneal (Icodextrin 7.5\%) solution as a last fill of 1.71 . He also received an Opti exchange of 2.01 of $1.36 \%$ solution in the evenings.

He was clinically and haemodynamically stable with mild abdominal discomfort as his symptom. Serum inflammatory markers showed a white cell count [WCC] of $6.0 \times 10^{6} / \mathrm{L}$ and a C-reactive protein [CRP] of 80.7 $\mathrm{mg} / \mathrm{L}$ (normal range $<5 \mathrm{mg} / \mathrm{L}$ ). Microscopy of the PD fluid showed a WCC of $155 \times 10^{6}$ cells/L and a total eosinophil count of $1 \times 10^{6}$ cells/L, but the Gram stain was negative. Empirical broad-spectrum intra-peritoneal antibiotics (Vancomycin and Gentamicin) were commenced. On day 5 , he developed worsening abdominal pain. On examination, his blood pressure was $158 / 100$ mmHg; heart rate $90 / \mathrm{min}$, temperature $37.5^{\circ} \mathrm{C}$. His abdomen was tender on palpation. There was no evidence of PD catheter exit site infection or any tunnel infection. The CRP was $456.9 \mathrm{mg} / \mathrm{L}$, and WCC was $12.3 \times 10^{6} / \mathrm{L}$. $\mathrm{He}$ was admitted to the renal unit and treatment was escalated to intravenous Gentamicin and Vancomycin, and subsequently to intravenous Meropenem on day 7 in view of persistent symptoms and raised inflammatory markers. He underwent rapid PD fluid exchanges to help relieve abdominal pain. On day 8 , his CRP was $534 \mathrm{mg} / \mathrm{L}$, WCC was $20.5 \times 10^{6} / \mathrm{L}$ with a temperature of $39.8^{\circ} \mathrm{C}$. He underwent emergency surgical PD catheter removal with peritoneal washout. Intra-operative findings did not reveal any bowel perforation. A haemodialysis catheter was inserted, and regular haemodialysis (HD) was commenced. On day 8 , acid-fast bacilli $[\mathrm{AFB}]$ were grown from the PD fluid sample, which was confirmed by the National Mycobacterium Reference Laboratory through whole-genome sequencing to be Mycobacterium abscessus. In view of this, he was commenced on quadruple therapy with intravenous Amikacin, Clarithromycin, Tigecycline and Imipenem with cilastatin. He developed new QTc prolongation on the electrocardiogram (ECG) on day 16, which was attributed to Clarithromycin, so this was therefore discontinued. Furthermore, he developed acute hepatic impairment which resolved following cessation of Tigecycline on day 26.

He remained clinically well on dual therapy with intravenous Amikacin and Imipenem and was discharged on day 35 (Fig. 1). A peripherally inserted central venous catheter was placed, to facilitate daily intravenous antibiotics as an outpatient. He continued on antibiotics for a total of 5 months. Linezolid was added during the last 6 weeks of therapy. He had a regular review by the Audiologists, but in spite of close monitoring to maintain the Amikacin levels within the therapeutic range, he has sustained a degree of sensorineural hearing loss.

\section{Discussion and conclusion}

M.abscessus is a nontuberculous mycobacterium (NTM). It is one of the most clinically relevant, rapidly growing mycobacteria, which are environmental organisms that usually grow in culture within 1 week. M. abscessus has been frequently isolated from water, soil, domestic and wild animals [6]. Although not highly virulent, M.abscessus is known to cause disseminated infection in immunocompromised hosts, and bacteraemia can occur in the context of dialysis catheter use [7]. It is a well-documented cause of pulmonary infection in patients with structural lung disease such as cystic fibrosis, and can cause skin and soft tissue infections in hospitalised post-surgical patients $[8,9]$.

\section{Diagnosis}

The diagnosis of NTM and M.abscessus PD peritonitis can be challenging. NTM can cause culture-negative infection, and it is interesting to note that in 4 out of 16 reported cases, the development of PD peritonitis was preceded by catheter replacement for the management of what was deemed to be culture-negative ESI or TI [3, 10, 11]. Clinicians should, therefore be vigilant with regards to possible NTM infection in patients with culture-negative PD peritonitis, particularly in the context of non-responsiveness to standard anti-microbial therapy $[5,12]$. 


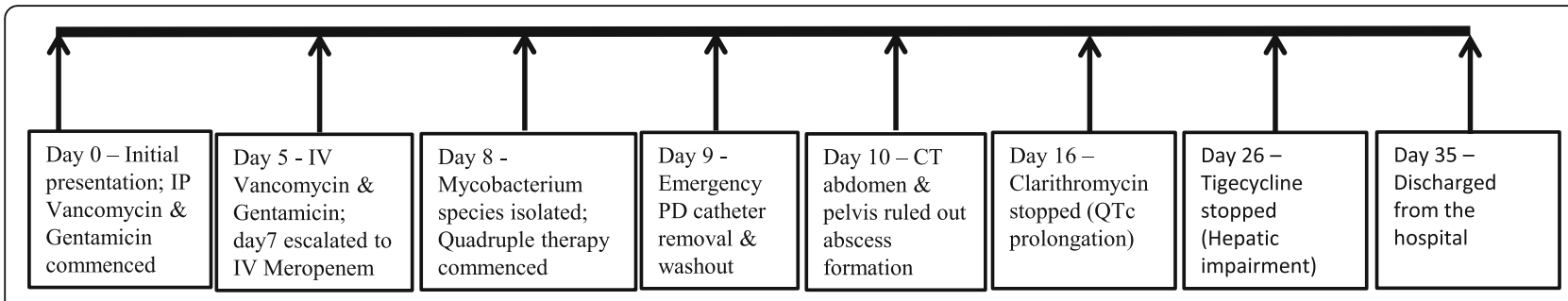

Fig. 1 Timeline of inpatient stay

Our patient was diabetic and on PD for his kidney failure. The presence of end-stage renal failure (ESRF) is associated with impairment of the innate and adaptive immune system [13]. Diabetes mellitus was present in $27.5 \%$ of cases of NTM related PD peritonitis in one systematic review, suggesting that metabolic disease may pre-dispose to NTM infection [5]. M.abscessus has a predisposition to create biofilms, colonise and infect catheters [7]. Consequently, all previously documented cases of M.abscessus PD peritonitis have required catheter removal for effective source control (Table 1). The intrinsic resistance of M.abscessus to classical antituberculous drugs as well as most other available broadspectrum antibiotics limits the choice of options for therapy [20] and poses a significant challenge with regards to treatment.

A systematic review of 57 cases of NTM PD peritonitis found that the time from onset of symptoms to the initiation of appropriate treatment was 4 weeks [5]. This delay is multi-factorial. Firstly, the available data do not demonstrate any factor unique to M.abscessus PD peritonitis compared with more common organisms. The symptoms and signs are indistinguishable from conventional PD peritonitis or tuberculous peritonitis [4, 21]. All cases of M.abscessus PD peritonitis first received empirical antibiotics and in one case, subsequently received anti-tuberculous therapy following a positive acid-fast bacillus (AFB) stain [22]. Secondly, the utility of AFB staining in such cases is debatable, as the sensitivity of this test is not high enough [23]. In one particular study, AFB staining was positive in only four out of 10 cases of M.abscessus peritonitis [4]. Most centres do not perform AFB staining routinely as it does not distinguish between tuberculous mycobacteria and NTM, thus providing no further information regarding anti-microbial sensitivities $[24,25]$. As a consequence, the diagnosis is reliant on bacterial culture alone, which leads to delays in the initiation of appropriate treatment and may likely result in worse outcome in such cases [26].

\section{Treatment}

The management of M.abscessus PD peritonitis is complex. Catheter removal appears to be essential to recovery in the acute setting, with long term management reliant on anti-microbial therapy $[12,17]$. M.abscessus is a multi-drug resistant organism, and as a consequence, the antibiotic choices are limited. One report investigated resistance and susceptibility criteria of M.abscessus in various published cases and showed that resistance rates for Quinolones, Doxycycline and Imipenem, were considerably high, while resistance rates for Cefoxitin (15.1\%) and Amikacin (7.7\%) were low. Clarithromycin showed variable resistance rates ranging from 0 to $38 \%$. As a consequence, treatment should be based on the susceptibility profile of the bacterium isolated from individual patients [18].

It has been recommended that non-pulmonary M.abscessus infection should be treated for between 4 and 6 months [27]. There is no consensus, however, for the optimal duration of the treatment for M.abscessus PD peritonitis and this is reflected in the variability of treatment regimens observed in the reported cases, where treatment duration ranged from 7 weeks to 28 weeks with a variety of drug combinations used based on individual sensitivities.

The challenges posed by the administration of longterm antibiotics should also be recognised. In our case, quadruple therapy was not tolerated due to the development of hepatic impairment with Tigecycline and QTc prolongation with Clarithromycin. Similar complications were observed in previous cases with Amikacin (sensorineural hearing loss) and Clarithromycin (hepatic impairment) [12]. The optimal duration of anti-microbial therapy is unclear at present but is likely to be at least 4 months [27].

Overall, outcomes from M.abscessus PD peritonitis appear to be worse than for conventional organisms. A 10year retrospective cohort study of PD peritonitis in an Australian population found that the risk of catheter removal varied between $<20$ to $>40 \%$ depending on the causative organism [17]. The risk of changing over to HD was estimated to be between 5 to $20 \%$ [1]. In previously reported cases of M.abscessus PD peritonitis, $100 \%$ of patients were converted to HD [6]. In only two of these cases were the patients successfully converted back to $\mathrm{PD}$, suggesting that the risk of PD failure is higher with M.abscessus infection compared with conventional organisms. 


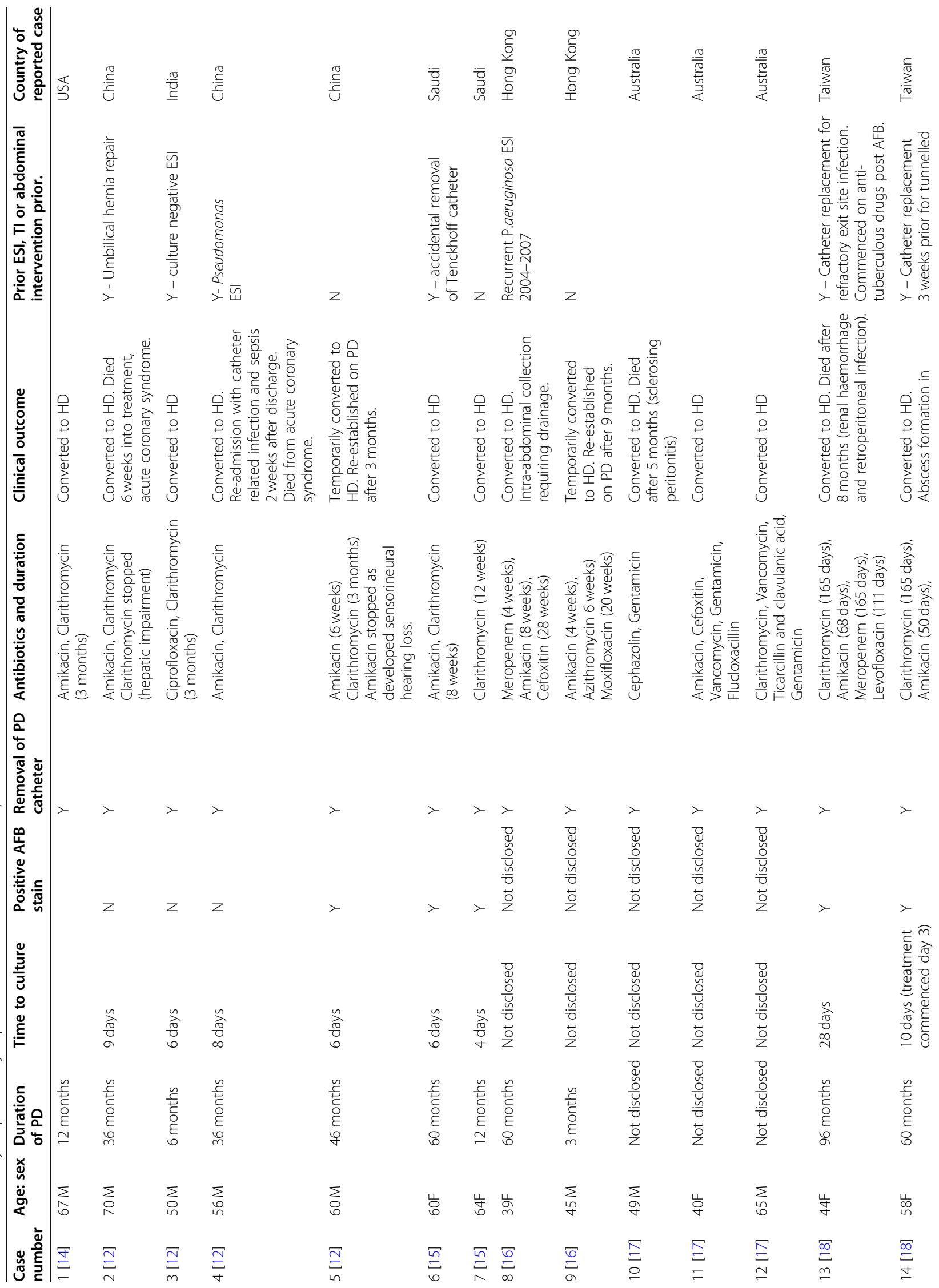




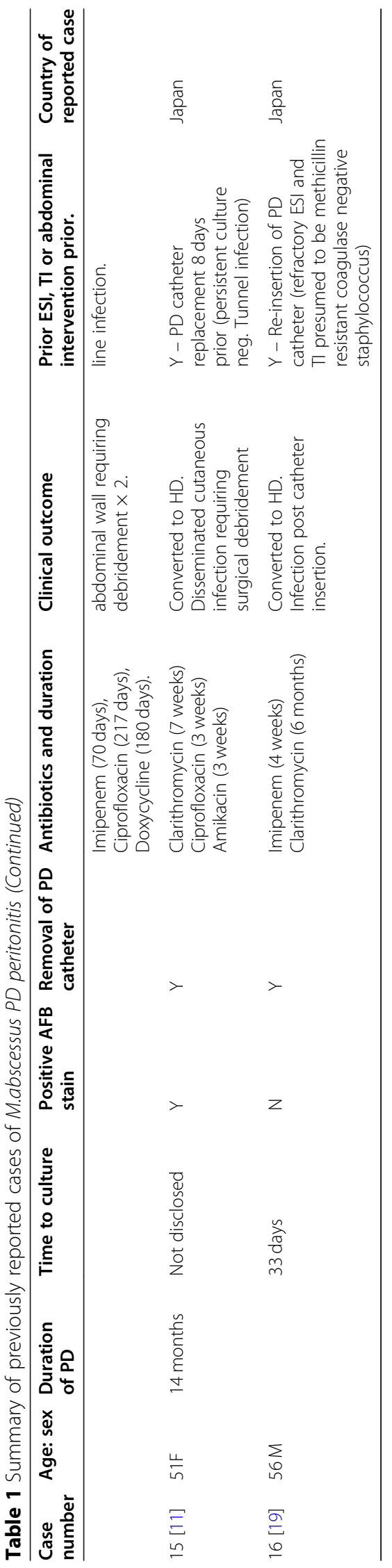


In summary, we present, to our knowledge, the first reported case of M.abscessus PD peritonitis in the United Kingdom. It was managed with catheter removal, a prolonged course of intravenous broad-spectrum antibiotics and conversion to HD. Clinicians should have a high index of suspicion of M.abscessus in cases of nonresolving PD related infections, or culture-negative peritonitis. Catheter removal appears to be essential for clinical recovery, and the switch to HD may be permanent.

\section{Abbreviations}

AFB: Acid-fast bacillus; CRP: C-reactive protein; ECG: Electrocardiogram; ESI: Exit-site infection; ESKD: End-stage kidney disease; HD: Haemodialysis; M abscessus: Mycobacterium abscessus; M.tuberculosis: Mycobacterium tuberculosis; M.leprae: Mycobacterium leprae; NTM: Nontuberculous mycobacteria; PD: Peritoneal dialysis; TI: Tunnel infection; UK: United Kingdom; WCC: White cell count

\section{Acknowledgements}

none.

\section{Authors' contributions}

Authors have provided substantial contributions to the concept, design of the work; the acquisition, and analysis, interpretation of data and the contributions to the drafting of the original manuscript and its subsequent revisions. All authors have read and have approved the submitted version and any substantially modified version that involves the author's contribution to the document. The authors have agreed to be personally accountable for the author's contributions and to ensure that the questions related to the accuracy or integrity of any part of the work, even ones in which the authors are not personally involved, are appropriately investigated, resolved, and the resolution documented in the literature. The individual author's contributions are declared below:- ASJ - looked after the patient, substantially contributed to the acquisition, analysis of the data and the preparation of the manuscript. JR - contributed substantially to the analysis of the data, design and the preparation of the manuscript. JC - contributed substantially to the analysis of the data, design and the preparation of the manuscript. DM - Looked after the patient, substantially contributed to the acquisition, analysis and interpretation of the data, in addition to the preparation of the manuscript. SS- Looked after the patient. Substantial contribution to the acquisition and interpretation of the data, design and concept of the manuscript.

\section{Funding}

Not applicable.

\section{Availability of data and materials}

Not applicable.

Ethics approval and consent to participate

Not applicable.

\section{Consent for publication}

Written informed consent for the publication of their clinical details and/or clinical images was obtained from the patient. A copy of the consent form is available for review by the Editor of this journal. The same has been uploaded on the submission site.

\section{Competing interests}

The authors declare no competing interests.

\section{Author details}

'South West Thames Renal \& Transplantation Unit, Epsom \& St Helier University Hospitals NHS Trust, Wrythe Lane, Carshalton, Surrey SM5 1AA, UK. ${ }^{2}$ Department of Microbiology, Epsom \& St Helier University Hospitals NHS Trust, Wrythe Lane, Carshalton, Surrey SM5 1AA, UK.
Received: 27 July 2020 Accepted: 2 November 2020

Published online: 17 November 2020

\section{References}

1. Htay H, Cho Y, Pascoe EM, Darssan D, Nadeau-Fredette AC, Hawley C, et al. Center effects and peritoneal Dialysis peritonitis outcomes: analysis of a National Registry. Am J Kidney Dis. 2018;71(6):814-21.

2. Li PKT, Szeto CC, Piraino B, de Arteaga J, Fan S, Figueiredo AE, et al. ISPD peritonitis recommendations: 2016 update on prevention and treatment. Peritoneal Dialysis Int Multimed Inc. 2016;36:481-508.

3. Rohit A, Abraham G. Peritoneal dialysis related peritonitis due to mycobacterium Spp.: a case report and review of literature. J Epidemiol Global Health Elsevier Ltd. 2016;6:243-8 [cited 2020 May 3] Available from: https://pubmed.ncbi.nlm.nih.gov/27443487/.

4. Karayaylali I, Seyrek N, Akpolat T, Ateş K, Ozener C, Yilmaz ME, et al. The prevalence and clinical features of tuberculous peritonitis in CAPD patients in Turkey, report of ten cases from multi-centers. Ren Fail. 2003;25(5):819-27.

5. Song Y, Wu J, Yan H, Chen J. Peritoneal dialysis-associated nontuberculous mycobacterium peritonitis: a systematic review of reported cases. Nephrol Dial Transplant. 2012;27(4):1639-44 Available from: http://10.0.4.69/ndt/gfr504.

6. Ding LW, Lai CC, Lee LN, Hsueh PR. Abdominal nontuberculous mycobacterial infection in a University Hospital in Taiwan from 1997 to 2003. J Formos Med Assoc. 2006;105(5):370-6 [cited 2020 Mar 8] Available from: https://www.sciencedirect.com/science/article/pii/S0929664609601327.

7. El Helou G, Viola GM, Hachem R, Han XY, Raad II. Rapidly growing mycobacterial bloodstream infections. Lancet Infect Dis. 2013;13:166-74.

8. Broda A, Jebbari H, Beaton K, Mitchell S, Drobniewski F. Comparative drug resistance of Mycobacterium abscessus and $\mathrm{M}$. chelonae isolates from patients with and without cystic fibrosis in the United Kingdom. J Clin Microbiol. 2013:51(1):217-23.

9. Shields RK, Clancy CJ, Minces LR, Shigemura N, Kwak EJ, Silveira FP, et al. Epidemiology and outcomes of deep surgical site infections following lung transplantation. Am J Transplant. 2013;13(8):2137-45.

10. Mooren VHJF, Bleeker MWP, van Ingen J, Hermans MHA, Wever PC. Disseminated mycobacterium abscessus infection in a peritoneal dialysis patient. IDCases. 2017;9:6-7.

11. Kameyama H, Mori Y, Kimura T, Sugishita C, Adachi T, Sonomura K, et al. A case report of mycobacterium abscessus peritonitis in a peritoneal dialysis patient. Ther Apher Dial. 2007;11(6):449-51.

12. Renaud CJ, Subramanian S, Tambyah PA, Lee EJC. The clinical course of rapidly growing nontuberculous mycobacterial peritoneal dialysis infections in Asians: a case series and literature review. Nephrology. 2011;16(2):174-9.

13. Kato S, Chmielewski M, Honda H, Pecoits-Filho R, Matsuo S, Yuzawa Y, et al. Aspects of immune dysfunction in end-stage renal disease. Clin J Am Soc Nephrol. 2008;3:1526-33.

14. Slagle K, Oblack D. Mycobacterium abscessus peritonitis: a case report. Clin Lab Sci. 1998:206-8 [cited 2020 May 3] Available from: https://pubmed.ncbi. nlm.nih.gov/10182108/.

15. Siddiqi N, Sheikh I. Peritonitis caused by mycobacterium abscesses in patients on continuous ambulatory peritoneal dialysis. Saudi J Kidney Dis Transpl. 2012:321-4 [cited 2020 May 3] Available from: https://pubmed.ncbi. nlm.nih.gov/22382227/.

16. Lo MW, Mak SK, Wong YY, Lo KC, Chan SF, Tong GMW, et al. Atypical mycobacterial exit-site infection and peritonitis in peritoneal dialysis patients on prophylactic exit-site gentamicin cream. Perit Dial Int. 2013; 33(3):267-72.

17. Jiang $\mathrm{SH}$, Roberts DM, Clayton PA, Jardine M. Nontuberculous mycobacterial PD peritonitis in Australia. Int Urol Nephrol. 2013;45(5):1423-8.

18. Yang TK, Lee JJ, Lu PL, Kuo HT, Kuo MC, Chen HC. Peritoneal dialysisassociated peritonitis caused by mycobacterium abscessus. Perit Dial Int. 2015;35(3):369-71 [cited 2020 May 3] Available from: https://pubmed.ncbi. nlm.nih.gov/26015424/.

19. Yoshimura R, Kawanishi M, Fujii S, Yamauchi A, Takase K, Yoshikane K, et al. Peritoneal dialysis-associated infection caused by Mycobacterium abscessus: a case report. BMC Nephrol. 2018;19(1):341 [cited 2020 May 3] Available from: https://bmcnephrol.biomedcentral.com/articles/10.1186/s12882-01 8-1148-2.

20. Nessar R, Cambau E, Reyrat JM, Murray A, Gicquel B. Mycobacterium abscessus: a new antibiotic nightmare. J Antimicrob Chemother. 2012;67(4): 810-8 Available from: http://10.0.4.69/jac/dkr578. 
21. Ram R, Swarnalatha G, Akpolat T, Dakshinamurty KV. Mycobacterium tuberculous peritonitis in CAPD patients: a report of 11 patients and review of literature. Int Urol Nephrol. 2013;45:1129-35.

22. Yang T-K, Lee J-J, Lu P-L, Kuo H-T, Kuo M-C, Chen H-C. Peritoneal dialysisassociated peritonitis caused by mycobacterium abscessus. 2015;35(3):369371. Available from: http://10.0.14.163/pdi.2014.00012.

23. Caulfield AJ, Wengenack NL. Diagnosis of active tuberculosis disease: from microscopy to molecular techniques. I Clin Tuberculosis Other Mycobacterial Dis Elsevier Ltd. 2016;4:33-43.

24. Chakravorty S, Sen MK, Tyagi JS. Diagnosis of extrapulmonary tuberculosis by smear, culture, and PCR using universal sample processing technology. J Clin Microbiol. 2005;43(9):4357-62 [cited 2020 Jul 4] Available from: /pmc/ articles/PMC1234147/?report=abstract.

25. Shu CC, Wang JT, Wang JY, Yu CJ, Lee LN. Mycobacterial peritonitis: difference between nontuberculous mycobacteria and Mycobacterium tuberculosis. Clin Microbiol Infect. 2012;18(3):246-52.

26. Esther CR, Hoberman S, Fine J, Allen S, Culbreath K, Rodino K, et al. Detection of rapidly growing mycobacteria in routine cultures of samples from patients with cystic fibrosis. J Clin Microbiol. 2011;49(4):1421-5 [cited $2020 \mathrm{Jul}$ 4] Available from: http://jcm.asm.org/.

27. Griffith DE, Aksamit T, Brown-Elliott BA, Catanzaro A, Daley C, Gordin F, et al. An official ATS/IDSA statement: diagnosis, treatment, and prevention of nontuberculous mycobacterial diseases. Am J Respir Crit Care Med. 2007; 175(4):367-416 Available from: https://pubmed.ncbi.nIm.nih.gov/17277290/.

\section{Publisher's Note}

Springer Nature remains neutral with regard to jurisdictional claims in published maps and institutional affiliations.

Ready to submit your research? Choose BMC and benefit from:

- fast, convenient online submission

- thorough peer review by experienced researchers in your field

- rapid publication on acceptance

- support for research data, including large and complex data types

- gold Open Access which fosters wider collaboration and increased citations

- maximum visibility for your research: over $100 \mathrm{M}$ website views per year

At $\mathrm{BMC}$, research is always in progress.

Learn more biomedcentral.com/submissions 